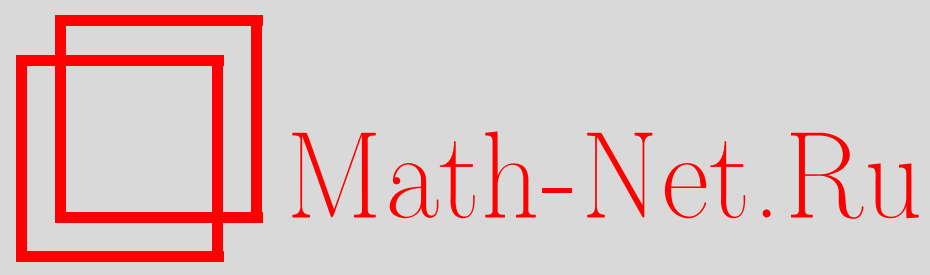

И. Р. Нежметдинов, Об однолистности одного интеграла на подклассах мероморфных функций, Матем. заметки, 2001, том 69, выпуск 1, 92-99

DOI: https://doi.org/10.4213/mzm486

Использование Общероссийского математического портала Math-Net.Ru подразумевает, что вы прочитали и согласны с пользовательским соглашением http://www . mathnet.ru/rus/agreement

Параметры загрузки:

IP: 3.89 .197 .203

26 апреля 2023 г., 15:54:10

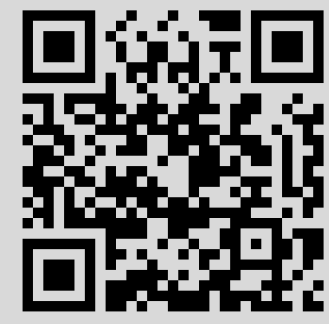




\section{ОБ ОДНОЛИСТНОСТИ ОДНОГО ИНТЕГРАЛА \\ НА ПОДКЛАССАХ МЕРОМОРФНЫХ ФУНКЦИЙ}

\section{И.Р. Нежметдинов}

Исследуется интегральный оператор $P_{\lambda}[f](\zeta)=\int_{\zeta_{0}}^{\zeta}\left(f^{\prime}(t)\right)^{\lambda} d t,|\zeta|>1$, действуюший на классе $\Sigma$ функций, мероморфных и однолистных во внешности единичного круга. Уточняются области значений параметра $\lambda$, при которых данный оператор сохраняет однолистность на $\Sigma$ или на его подклассе, состоящем из выпуклых функций. Как следствие получается двусторонняя оценка разделяющей постоянной в достаточном условии однолистной разрешимости внешней обратной краевой задачи.

Библиографофия: 8 названий.

Пусть $\Sigma$ - класс функций вида $f(\zeta)=\zeta+\sum_{k=0}^{\infty} \alpha_{k} \zeta^{-k}$, регулярных и однолистных в области $E^{-}=\{\zeta: 1<|\zeta|<\infty\}$ и имеющих простой полюс в $\zeta=\infty$. Рассмотрим интегральньй оператор

$$
P_{\lambda}[f](\zeta)=\int_{\zeta_{0}}^{\zeta}\left(f^{\prime}(t)\right)^{\lambda} d t
$$

где $\lambda \in \mathbb{C}, \zeta_{0} \in E^{-}$.

Обозначим через $\Lambda(\mathfrak{M}, \Sigma)$ множество всех таких $\lambda$, что $P_{\lambda}[\mathfrak{M}] \subset \Sigma$, где $\mathfrak{M}=\Sigma$ или $\Sigma^{0}\left(\Sigma^{0}-\right.$ подкласс $\Sigma$, состоящий из выпуклых функций [1, с. 10]).

Проблема определения аналогичных множеств для оператора

$$
P_{\lambda, 0}[f](z)=\int_{0}^{z}\left(f^{\prime}(t)\right)^{\lambda} d t, \quad|z|<1,
$$

действующего на классе $S$ функций, однолистньх в единичном круге, хорошо известна (см. работы [2]-[4]). Полагая $\Lambda(\mathfrak{N}, S)=\left\{\lambda \in \mathbb{C}: P_{\lambda, 0}[\mathfrak{N}] \subset S\right\}$, запишем имеюшиеся результаты в виде

$$
\Lambda\left(S^{0}, S\right)=\left\{\lambda:|\lambda| \leqslant \frac{1}{2}\right\} \cup\left\{\lambda: \frac{1}{2} \leqslant \lambda \leqslant \frac{3}{2}\right\},
$$

где $S^{0}-$ подкласс вьпуклых функций и

$$
\left\{\lambda:|\lambda| \leqslant \frac{1}{4}\right\} \subset \Lambda(S, S) \subset\left\{\lambda:|\lambda| \leqslant \frac{1}{3}\right\} \cup\{1\} .
$$

Для дальнейшего понадобится достаточное условие однолистности, принадлежашее И. Беккеру [5]. 
ЛЕмма 1. Пусть функиия $f(\zeta)=\zeta+\sum_{k=0}^{\infty} \alpha_{k} \zeta^{-k}$ регулярна в $E^{-}$и при некотором $c \neq 1,|c| \leqslant 1$, удовлетворяет условию

$$
\left|\frac{\left(|\zeta|^{2}-c\right)\left(|\zeta|^{2}-1\right) \zeta f^{\prime \prime}(\zeta)}{(1-c) f^{\prime}(\zeta)}+c\right| \leqslant|\zeta|^{2}, \quad \zeta \in E^{-}
$$

Тогда $f(\zeta)$ однолистна в $E^{-}$.

Теорема 1. Справедливы включения

$$
\left\{\lambda:|\lambda| \leqslant \frac{1}{4}\right\} \subset \Lambda(\Sigma, \Sigma) \subset\left\{\lambda:|\lambda|+\frac{|\lambda-1|}{2} \leqslant 1\right\} .
$$

ДокАЗАТЕЛЬСтво. Пусть $F=P_{\lambda}[f]$, где $f \in \Sigma$. Повторно дифференцируя соотношение (1), получим

$$
\frac{\zeta F^{\prime \prime}(\zeta)}{F^{\prime}(\zeta)}=\frac{\lambda \zeta f^{\prime \prime}(\zeta)}{f^{\prime}(\zeta)}, \quad \zeta \in E^{-}
$$

Известно (см. [6, с. 139]), что на классе $\Sigma$ имеет место точная оценка

$$
\left|\frac{\zeta f^{\prime \prime}(\zeta)}{f^{\prime}(\zeta)}+\frac{4 \varkappa(\rho)-2}{\rho-1}\right| \leqslant \frac{4 \varkappa(\rho)}{\rho-1}, \quad \rho=|\zeta|^{2}>1,
$$

где функция $\varkappa(\rho)=\rho\left(1-E\left(\rho^{-1 / 2}\right) / K\left(\rho^{-1 / 2}\right)\right)$ выражена через полные эллиптические интегралы

$$
K(m)=\int_{0}^{\pi / 2} \frac{d \theta}{\sqrt{1-m^{2} \sin ^{2} \theta}}, \quad E(m)=\int_{0}^{\pi / 2} \sqrt{1-m^{2} \sin ^{2} \theta} d \theta
$$

Если $|\lambda| \leqslant 1 / 4$, то при $\zeta \in E^{-}$из $(2)$ и (3) получим

$$
\left|\frac{\zeta F^{\prime \prime}(\zeta)}{F^{\prime}(\zeta)}+\frac{2 \lambda(1-2 \lambda)}{(\rho-1)(\rho-2 \lambda)}\right| \leqslant \frac{1}{\rho-1}\left(\varkappa(\rho)+\left|\frac{1}{2}-\varkappa(\rho)+\frac{1-2 \lambda}{2(\rho-2 \lambda)}\right|\right) .
$$

В силу (4) и леммы 1 (при $c=2 \lambda$ ) функция $F(\zeta)$ будет однолистной, если

$$
G(w) \equiv \varkappa(\rho)|w|+\left|\left(\frac{1}{2}-\varkappa(\rho)\right) w+\frac{1}{2}\right| \leqslant \rho,
$$

причем область значений переменной $w=(\rho-2 \lambda) /(1-2 \lambda)$ есть замкнутьй круг $|w-a| \leqslant b$, где $a=(4 \rho-1) / 3, b=2(\rho-1) / 3$. Функция $G(w)$ достигает максимума на границе этого круга (достаточно заметить, что $\partial G / \partial v=0$, где $v=\operatorname{Im} w$, только при $v=0$, но там максимума, очевидно, нет). Теперь, полагая $w=a+b e^{i \psi}, 0 \leqslant \psi \leqslant 2 \pi$, будем иметь

$$
\begin{aligned}
G(w) & =\sqrt{(1 / 2-\sigma a)^{2}+b^{2} \sigma^{2}-2 b \sigma(1 / 2-a \sigma) \cos \psi}+\varkappa(\rho) \sqrt{a^{2}+b^{2}+2 a b \cos \psi} \\
& \equiv G_{1}(\cos \psi)
\end{aligned}
$$

где $\sigma=\varkappa(\rho)-1 / 2>0($ см. [7]). 
Заметим, что $G_{1}^{\prime \prime}(t) \leqslant 0$, откуда $G_{1}^{\prime}(t) \geqslant G_{1}^{\prime}(1)$ при $-1 \leqslant t \leqslant 1$. Остается проверить, что $G_{1}^{\prime}(1) \geqslant 0$, поскольку тогда $G_{1}(t) \leqslant G_{1}(1)=\rho$ и неравенство $(5)$ вьполняется.

С этой целью докажем оценку

$$
\varkappa(\rho) \leqslant \frac{1}{2}+\frac{4 \rho-1}{2\left(8 \rho^{2}-4 \rho-1\right)}, \quad \rho>1 .
$$

Используя формулы (5.2.16.6) и (5.2.16.7) из [8], дающие разложения функций $K(m)$, $E(m)$ в ряды Маклорена, получаем

$$
\varkappa(\rho)=\frac{\sum_{k=0}^{\infty} c_{k}(2 k+1) \rho^{-k} /(2 k+2)}{\sum_{k=0}^{\infty} c_{k} \rho^{-k}}, \quad \text { где } \quad c_{k}=\left(\frac{(2 k-1) ! !}{(2 k) ! !}\right)^{2}, \quad k \geqslant 0
$$

(при этом полагаем $(-1) ! !=0 ! !=1)$.

Оценка (7) приводится к виду

$$
\left(8 \rho^{2}-4 \rho-1\right) \sum_{k=0}^{\infty} k(k+1)^{-1} c_{k} \rho^{-k} \leqslant(4 \rho-1) \sum_{k=0}^{\infty} c_{k} \rho^{-k}
$$

откуда после элементарных преобразований получаем

$$
3 \rho-\frac{1}{4}+\sum_{k=1}^{\infty}\left(\frac{4(2 k+3)}{k+2} c_{k+1}-\frac{8(k+2)}{k+3} c_{k+2}-\frac{c_{k}}{k+1}\right) \rho^{-k} \geqslant 0, \quad \rho>1 .
$$

Теперь достаточно заметить, что при $k \geqslant 1$ имеем

$$
\begin{aligned}
& \frac{4(2 k+3)}{k+2} c_{k+1}-\frac{8(k+2)}{k+3} c_{k+2}-\frac{c_{k}}{k+1} \\
& =\frac{c_{k}}{k+1}\left(\frac{3(2 k+1)^{2}(2 k+3)}{2(k+1)(k+2)(k+3)}-1\right)>0
\end{aligned}
$$

и неравенство (8) (а, следовательно, и (7)) доказано.

Отсюда имеем

$$
G_{1}^{\prime}(1)=\frac{(\rho-1)\left(4 \rho-1-2\left(8 \rho^{2}-4 \rho-1\right) \sigma\right)}{9(2 \rho-1)(1-2(2 \rho-1) \sigma)} \geqslant 0
$$

что и требовалось.

Докажем вторую часть теоремы. Из свойств функций $K(m), E(m)$ следует, что $\varkappa(\rho) \rightarrow 1$ при $\rho \rightarrow 1$. Если $|\lambda|+|\lambda-1| / 2>1$, то найдется $\rho_{0}>1$ такое, что

$$
\left(4 \varkappa\left(\rho_{0}\right)-2\right)|1-\lambda|+4|\lambda| \varkappa\left(\rho_{0}\right)>4 \varkappa\left(\rho_{0}\right) .
$$

Подберем вешественное $\alpha$, для которого

$$
\left(4 \varkappa\left(\rho_{0}\right)-2\right)|1-\lambda|+4|\lambda| \varkappa\left(\rho_{0}\right)=\left|\left(4 \varkappa\left(\rho_{0}\right)-2\right)(1-\lambda)+4 \lambda e^{i \alpha} \varkappa\left(\rho_{0}\right)\right| .
$$


Оценка (3) характеризует область значений функционала $\zeta f^{\prime \prime}(\zeta) / f^{\prime}(\zeta)$ на классе $\Sigma$ ( $\zeta \in E^{-}$фиксированное). Поэтому найдутся $f_{0} \in \Sigma$ и $\zeta_{0}^{\prime} \in E^{-}$такие, что

$$
\frac{\zeta_{0}^{\prime} f_{0}^{\prime \prime}\left(\zeta_{0}^{\prime}\right)}{f_{0}^{\prime}\left(\zeta_{0}^{\prime}\right)}=\frac{2-4 \varkappa\left(\rho_{0}\right)+4 \varkappa\left(\rho_{0}\right) e^{i \alpha}}{\rho_{0}-1}, \quad\left|\zeta_{0}^{\prime}\right|^{2}=\rho_{0}
$$

Теперь если $F_{0}=P_{\lambda}\left[f_{0}\right]$, то в силу $(2)$ и (9)-(11) будем иметь

$$
\left|\frac{\zeta_{0} F_{0}^{\prime \prime}\left(\zeta_{0}\right)}{F_{0}^{\prime}\left(\zeta_{0}\right)}+\frac{4 \varkappa\left(\rho_{0}\right)-2}{\rho_{0}-1}\right|>\frac{4 \varkappa\left(\rho_{0}\right)}{\rho_{0}-1},
$$

откуда $F_{0} \notin \Sigma$.

Теорема 1 уточняет результат из [4], согласно которому

$$
\left\{\lambda:|\lambda| \leqslant \frac{1}{6}\right\} \subset \Lambda(\Sigma, \Sigma) \subset\{\lambda:|\lambda| \leqslant 1\} .
$$

ТЕорема 2. Имеет место включение

$$
\left\{\lambda=\mu e^{i \nu}:|\lambda| \leqslant \mu_{0}(\nu)\right\} \subset \Lambda\left(\Sigma^{0}, \Sigma\right)
$$

где $\mu_{0}(\nu)=(3+\cos \nu) / 4 n p u \nu_{0} \leqslant|\nu| \leqslant \pi u \mu_{0}(\nu)=(1+|\sin \nu|)^{-1} n p u 0 \leqslant|\nu| \leqslant \nu_{0}$. Здесь $\nu_{0}=\arccos t_{0}=0.9633 \ldots, a t_{0}=0.5707 \ldots-$ единственный корень уравнения $47-52 t-46 t^{2}-12 t^{3}-t^{4}=0$ в интервале $0<t<1$.

ДокаЗАТЕЛЬСтво. Достаточно проверить, что $\lambda \in \Lambda\left(\Sigma^{0}, \Sigma\right)$ при $|\lambda|=\mu=\mu_{0}(\nu)$. Действительно, множество $\Lambda\left(\Sigma^{0}, \Sigma\right)$ звездообразно относительно точки $\lambda=0$, т.е. из $\lambda \in \Lambda\left(\Sigma^{0}, \Sigma\right)$ следует, что $t \lambda \in \Lambda\left(\Sigma^{0}, \Sigma\right)$ при любом $0 \leqslant t \leqslant 1$.

Пусть

$$
\mu=|\lambda|=\frac{3+\cos \nu}{4}, \quad \nu_{0} \leqslant|\nu| \leqslant \pi
$$

Тогда, полагая

$$
c=1-2 \mu e^{i \theta}, \quad \theta=2 \operatorname{arctg}\left(\sin \nu(3+\cos \nu)^{-1}\right)
$$

находим

$$
c=\frac{-(1+\cos \nu)\left(2+5 \cos \nu+\cos ^{2} \nu\right)-i \sin \nu(3+\cos \nu)^{2}}{2(5+3 \cos \nu)} .
$$

Нетрудно видеть, что $|c| \leqslant 1$ тогда и только тогда, когда $\mu \leqslant \cos \theta$, а последнее неравенство всегда вьполнено в силу (12) и (13) .

Если $f \in \Sigma^{0}$, то из условия вьпуклости (см. [4]) получим

$$
\left|\frac{\zeta f^{\prime \prime}(\zeta)}{f^{\prime}(\zeta)}-\frac{2}{\rho^{2}-1}\right| \leqslant \frac{2 \rho}{\rho^{2}-1}, \quad \rho=|\zeta|^{2}>1 .
$$

Отсюда и из $(2)$ для $F=P_{\lambda}[f], \zeta \in E^{-}$, имеем

$$
\left|\frac{\zeta F^{\prime \prime}(\zeta)}{F^{\prime}(\zeta)}+\frac{c(1-c)}{\left(\rho^{2}-1\right)\left(\rho^{2}-c\right)}\right| \leqslant \frac{2 \mu \rho}{\rho^{2}-1}+\frac{1}{\rho-1}\left|\frac{2 \lambda}{\rho+1}+\frac{c(1-c)}{\rho-c}\right| .
$$


В силу (13), (14) и леммы 1 функция $F(\zeta)$ будет однолистной, если

$$
\left|(\rho-c) e^{i(\nu-\theta)}+c(\rho+1)\right| \leqslant \rho(\rho+1-|\rho-c|), \quad \rho>1 .
$$

Поскольку $|c| \leqslant 1$, то правая часть в (15) неотрицательна и возведение в квадрат этого неравенства будет равносильньм преобразованием. Следовательно, достаточно доказать, что

$$
\rho^{2}|\rho-c| \leqslant \rho^{3}-\rho^{2} \operatorname{Re} c+\rho \operatorname{Re}\left(\bar{c}\left(1-e^{i(\nu-\theta)}\right)\right)-|c|^{2}(1-\cos (\nu-\theta)) .
$$

При $\rho>1$ непосредственно получаем

$$
\begin{aligned}
& \rho^{3}-\rho^{2} \operatorname{Re} c \geqslant \rho^{2}(1-\operatorname{Re} c) \geqslant 0 ; \\
& \rho^{3}-\rho^{2} \operatorname{Re} c+\rho \operatorname{Re}\left(\bar{c}\left(1-e^{i(\nu-\theta)}\right)\right) \geqslant \rho\left(1-\operatorname{Re}\left(\bar{c}\left(1-e^{i(\nu-\theta)}\right)\right)\right) \geqslant 0 ; \\
& \rho^{3}-\rho^{2} \operatorname{Re} c+\rho \operatorname{Re}\left(\bar{c}\left(1-e^{i(\nu-\theta)}\right)\right)-|c|^{2}(1-\cos (\nu-\theta)) \\
& \quad \geqslant 1-|c|^{2}-\operatorname{Re}\left(\bar{c}(1-c) e^{i(\nu-\theta)}\right)=1+\mu^{2}-|\lambda+c|^{2}=0,
\end{aligned}
$$

поэтому неравенство (16) тоже можно возвести в квадрат.

Полагая для краткости $x=\cos \nu$, после обширных, но элементарных выкладок запишем

$$
a_{3} \rho^{3}+a_{2} \rho^{2}+a_{1} \rho+a_{0} \geqslant 0
$$

где

$$
\begin{aligned}
& a_{3}=(5+3 x)^{2}\left(47-52 x-46 x^{2}-12 x^{3}-x^{4}\right), \\
& a_{2}=115+974 x+1653 x^{2}+1036 x^{3}+277 x^{4}+38 x^{5}+3 x^{6}, \\
& a_{1}=799+476 x-761 x^{2}-504 x^{3}-43 x^{4}+28 x^{5}+5 x^{6}, \\
& a_{0}=-289+323 x^{2}-35 x^{4}+x^{6} .
\end{aligned}
$$

Очевидно, что при $-1 \leqslant x \leqslant 0$ имеем $a_{3}>0$, а на отрезке $0 \leqslant x \leqslant 1$ этот многочлен имеет единственный корень $x=t_{0}=0.5707 \ldots$ Отсюда при $-1 \leqslant x \leqslant t_{0}$ в силу цепочки неравенств

$$
\begin{aligned}
a_{3} & \geqslant 0, \\
a_{3}+a_{2}=2(5+3 x)(1-x)(3+x)\left(43+39 x+13 x^{2}+x^{3}\right) & \geqslant 0, \\
a_{3}+a_{2}+a_{1}=(1-x)\left(2089+3649 x+2254 x^{2}+638 x^{3}+73 x^{4}+x^{5}\right) & \geqslant 0, \\
a_{3}+a_{2}+a_{1}+a_{0}=8(1-x)\left(15+14 x+3 x^{2}\right)^{2} & \geqslant 0
\end{aligned}
$$

заключаем, что оценка (17) вьполнена при $\nu_{0} \leqslant|\nu| \leqslant \pi$.

Заметим, что в этом случае нельзя улучшить оценку для $|\lambda|$, используя лишь лемму 1 . Во второй части теоремы результат допускает уточнение, однако получить аналитическое выражение для оптимального $\mu$ при $|\nu| \leqslant \nu_{0}$ не представляется возможньм.

Доказательство второй части теоремы проведем аналогично. Сначала заметим, что для однолистности функции $F=P_{\lambda}[f]$ в силу неравенства (5) и леммы 1 достаточно выполнения условия

$$
H(\rho, \lambda, c) \equiv 2|\lambda||\rho-c|+\rho^{-1}|2 \lambda(\rho-c)+c(1-c)(\rho+1)| \leqslant|1-c|(\rho+1)
$$


при $\rho>1$. Подставляя сюда $c=-a, 0 \leqslant a \leqslant 1$, и $\lambda=a \pm i\left(1-a^{2}\right) / 2$, запишем это неравенство в виде

$$
|a(\rho-1)+(1+a)(\rho+a) i| \leqslant \rho\left(a \rho+1+a+a^{2}\right), \quad \rho>1 .
$$

После элементарных преобразований получим

$$
a^{2} \rho^{3}+a\left(2+3 a+2 a^{2}\right) \rho^{2}+a^{2}\left(2+4 a+a^{2}\right) \rho-a^{4} \geqslant 0, \quad \rho>1,
$$

что легко проверяется.

В статье [4] с помощью принципа площадей было доказано, что если $|\lambda|>1$, то $\lambda \notin \Lambda\left(\Sigma^{0}, \Sigma\right)$, однако, как будет видно из доказательства следующего утверждения, принцип площадей не дает здесь точной характеристики однолистности.

ТЕОРема 3. Пересечение множества $\Lambda\left(\Sigma^{0}, \Sigma\right)$ с вещественной осъю совпадает с отрезком $[-1 / 2,1]$.

ДокАЗАТЕЛЬСТво. С учетом теоремы 2 и предыдущего замечания достаточно показать, что при $\lambda<-1 / 2$ имеем $P_{\lambda}\left[\Sigma^{0}\right] \not \subset \Sigma$. Известно, что $f_{0}(\zeta)=\zeta+1 / \zeta \in \Sigma^{0}$, откуда

$$
\begin{aligned}
F(\zeta, \lambda) & =P_{\lambda}\left[f_{0}\right](\zeta)=\int_{\zeta_{0}}^{\zeta}\left(1-t^{-2}\right)^{\lambda} d t \\
& =\zeta+C+\sum_{k=1}^{\infty} \frac{\lambda(1-\lambda)(2-\lambda) \cdots(k-1-\lambda)}{k !(2 k-1) \zeta^{2 k-1}}
\end{aligned}
$$

Вместо $F(\zeta, \lambda)$ удобнее рассматривать функцию $\widetilde{F}(\zeta, \lambda)=F(\zeta, \lambda)-C$, которая является нечетной и удовлетворяет условию

$$
\overline{\widetilde{F}(\zeta, \lambda)}=\widetilde{F}(\bar{\zeta}, \lambda), \quad \zeta \in D^{-} .
$$

Отсюда следует, что отображение $\widetilde{F}(\zeta, \lambda)$ сохраняет симметрию относительно мнимой оси.

Нетрудно показать, что $w=\widetilde{F}(\zeta,-1 / 2)=\sqrt{\zeta^{2}-1}$ отображает область $E^{-}$на внешность лемнискаты

$$
\left\{w=\tau e^{i \chi}: \tau \geqslant \sqrt{-2 \cos 2 \chi},\left|\chi \pm \frac{\pi}{2}\right| \leqslant \frac{\pi}{4}\right\},
$$

причем $\widetilde{F}( \pm 1,-1 / 2)=0$. Из представления (18) следует, что при любом $\lambda<-1 / 2$ имеем $\widetilde{F}(1, \lambda)<\widetilde{F}(1,-1 / 2)=0$, откуда найдется $r_{0}>1$ такое, что $\widetilde{F}\left(r_{0}, \lambda\right)<0$.

С другой стороны, поскольку $\operatorname{Re} \widetilde{F}\left(e^{i \pi / 3},-1 / 2\right)=3^{1 / 4} \cos (5 \pi / 12)>0$, то при некоторых $\lambda<-1 / 2$ и $r_{0}>1$ будем иметь $\operatorname{Re} \widetilde{F}\left(r_{0} e^{i \pi / 3}, \lambda\right)>0$.

Следовательно, дуга $w=\widetilde{F}\left(e^{i \chi}\right), 0 \leqslant \chi \leqslant \pi / 3$, пересекает мнимую ось, а в силу отмеченной симметричности рассматриваемой функции и дугу $w=\widetilde{F}\left(e^{i \chi}\right), 2 \pi / 3 \leqslant \chi \leqslant \pi$. Отсюда функция $\widetilde{F}(\zeta, \lambda)$ неоднолистна в $E^{-}$. 
С помощью теоремы 2 можно улучшить оценку разделяющей постоянной в достаточном условии однолистной разрешимости внешней основной обратной краевой задачи (см. [1, с. 62]). Это решение определяется формулой

$$
f(\zeta)=e^{i \beta} \int_{\zeta_{0}}^{\zeta} \exp \left(-\frac{1}{2 \pi} \int_{0}^{2 \pi} p(\theta) \frac{e^{i \theta}+t}{e^{i \theta}-t} d \theta\right) d t+C, \quad \zeta \in E^{-}
$$

где $\beta, C$-постоянные, а $p(\theta)=\ln (d s / d \theta)$ - заданная функция, причем

$$
\int_{0}^{2 \pi} p(\theta) e^{i \theta} d \theta=0
$$

СлЕдСТВИЕ 1. Если 2л-периодическая дифференцируемая функиия $p(\theta)$ удовлетворяет условию разрешимости (20) и, кроме того,

$$
p^{\prime}(\theta) \geqslant-0.75 \quad\left(\text { uлu } \quad p^{\prime}(\theta) \leqslant 0.75\right)
$$

то функция (19) однолистна. При замене в условии (21) постоянной 0.75 на $0.893 \ldots$ найдутся неоднолистные решения обратной краевой задачи, удовлетворяющие ему.

ДокАЗАТЕЛьСтво. Достаточность условия (21) следует из теоремы 2 при $\nu= \pm \pi / 2$, поскольку $p^{\prime}(\theta)=\operatorname{Re} i e^{i \theta} f^{\prime \prime}\left(e^{i \theta}\right) / f^{\prime}\left(e^{i \theta}\right)$. С другой стороны, согласно теореме площадей функция $F(\zeta, \lambda)$, заданная формулой $(18)$, заведомо неоднолистна, если только

$$
H_{0}(\lambda) \equiv \sum_{k=1}^{\infty} \frac{|\lambda|^{2}|\lambda-1|^{2} \cdots|\lambda-k+1|^{2}}{(k !)^{2}(2 k-1)}>1
$$

При фиксированном натуральном $N$ имеем

$$
\begin{aligned}
H_{0}(i \mu) \geqslant & \sum_{k=1}^{N} \frac{\mu^{2}\left(\mu^{2}+1\right) \cdots\left[\mu^{2}+(k-1)^{2}\right]}{(k !)^{2}(2 k-1)} \\
& +\frac{\mu^{2}\left(\mu^{2}+1\right) \cdots\left(\mu^{2}+N^{2}\right)}{(N !)^{2}} \sum_{k=N+1}^{\infty} \frac{1}{k^{2}(2 k-1)} .
\end{aligned}
$$

Поскольку $\sum_{k=1}^{\infty} k^{-2}(2 k-1)^{-1}=4 \ln 2-\pi^{2} / 6=1.1276 \ldots$, а функция в правой части монотонно возрастает по $\mu$, заметим, что оценка для $\mu$, следующая из неравенств (22), (23), убывает с увеличением $N$. В частности, при $N=8$ получим, что $F(\zeta, i \mu)$ неоднолистна при $\mu>0.8935 \ldots$.

Тем самым улучшаются как верхняя, так и нижняя оценка разделяющей постоянной для достаточного условия однолистности $p^{\prime}(\theta) \geqslant-0.5$, полученного в [4]. 


\section{СПИСОК ЦИТИРОВАННОЙ ЛИТЕРАТУРЫ}

[1] Авхадиев Ф.Г., Аксентьев Л.А., Елизаров А. М. Достаточные условия конечнолистности аналитических функций и их приложения // Итоги науки и техники. Математический анализ. Т. 25. М.: ВИНИТИ, 1987. С. 3-121.

[2] Pfaltzgraff J. A. Univalence of the integral $\left(f^{\prime}(z)\right)^{\alpha} / /$ Bull. London Math. Soc. 1975. V. 7. № 3. P. 254-256.

[3] Royster W. C. On the univalence of a certain integral // Michigan Math. J. 1965. V. 12. P. $385-387$.

[4] Аксентьев Л.А., Нежметдинов И.Р. Достаточные условия однолистности некоторых интегральных представлений // Труды семин. по краевым задачам. Вып. 18. Казань: Изд-во КГУ, 1982. С. 3-11.

[5] Becker J. Conformal mappings with quasiconformal extensions // Aspects of Contemporary Complex Analysis. London: Academic Press, 1980. P. 37-72.

[6] Голузин Г. М. Геометрическая теория функций комплексного переменного. М.: Наука, 1966.

[7] Авхадиев Ф. Г. Об условиях однолистности аналитических функций // Изв. вузов. Матем. 1970. №11. С. 3-13.

[8] Прудников А. П., Брычков Ю. А., Маричев О. И. Интегралы и ряды. Элементарные функции. М.: Наука, 1981.

Казанский государственный университет

Поступило

E-mail : nezh@ksu.ru

20.09.1999 